\title{
Analysis of Heart Rate Variability Indices after Selective Acute Atrial Ischemia in Humans
}

\author{
Pedro Gomis ${ }^{1,2}$, Jesús Ávarez-García ${ }^{3}$, Pere Caminal ${ }^{1,2}$, Juan Cinca ${ }^{3}$ \\ ${ }^{1}$ ESAII Dept., EEBE, CREB, Universitat Politècnica de Catalunya, Barcelona, Spain \\ ${ }^{2}$ CIBER - BBN (Bioenginering, Biomedicine and Nanomedicine), Spain \\ ${ }^{3}$ Cardiology Dept., Hospital de la Santa Creu i Sant Pau, IIB-SantPau, Universidad Autónoma de \\ Barcelona, Barcelona, Spain
}

\begin{abstract}
The purpose of this work is to analyze the effect of selective atrial coronary artery occlusion on heart rate variability (HRV). Twelve-hour, Holter recording were studied in 109 patients undergoing elective percutaneous transluminal coronary angioplasty (PTCA) of the right or circumflex coronary artery. Accidental atrial branch occlusions $(A B O)$ were found in 17 patients at the end of the interventions. $R$ - $R$ intervals from sinus origin were used to calculate standard time and frequency domain and fractal HRV indices. Fractal properties were measured by the exponent $\alpha 1$, using detrended fluctuation analysis (DFA) of the $R-R$ series.

During the first 10 minutes immediately after PTCA, $S D N N$ was significantly lower in $A B O$ patients respect to non-ABO (32.5 \pm 16.0 vs. $49.5 \pm 28.6 \mathrm{~ms}, \quad p=0.016)$, showing a reduced autonomic response. Fractal index values $\alpha_{1}$ were similar in both groups. The 12-hour RR interval series showed an averaged $H R$ of $66 \pm 9 \mathrm{bpm}$ in patients with $A B O$ and $63 \pm 9$ bpm in patients without $A B O$ $(p=0.16)$, and no significant differences were found in the analyzed HRV indices. A higher incidence of atrial tachycardia (41\% vs. $15 \%, p=0.02)$ and atrial fibrillation (2 $A B O$ vs. $O$ non- $A B O, p=0.025$ ) was found in patients with $A B O$.

Acute atrial ischemia can lead to a global decrease in the autonomic response that recovers after several minutes and may be associated with atrial arrhythmias.
\end{abstract}

\section{Introduction}

Acute ventricular ischemia has been linked with arrhythmogenesis [1] and different vagal and sympathetic response according to the coronary artery occlusion [2]. However, little knowledge is available of the arrhythmogenic effects of acute atrial ischemia and its impact on the autonomic nervous system (ANS) response.
Atrial coronary arteries arise from the right and circumflex coronary arteries, thus they be affected or occluded during the angioplasty procedure of placing a stent in these arteries. Accidental occlusion of atrial coronary arteries, occurring during elective percutaneous transluminal coronary angioplasty (PTCA), may be an excellent clinical model for studying atrial ischemia in humans and its effects on cardiac arrhythmias [3,4]. The purpose of this study is to analyze, immediately after the PTCA procedures, the effect of selective atrial coronary artery occlusion on heart rate variability (HRV) and assess potential arrhythmia effects.

\section{Methods}

\subsection{Population and data acquisition}

From a prospective cohort study previously described [4], 109 patients undergoing elective PTCA of the right or circumflex coronary artery were chosen. Following the thrombolysis in myocardial infarction (TIMI) trial (see details in [4]), accidental atrial branch occlusions (ABO) were found in 17 patients at the end of the coronary intervention. The rest of the 92 patients were considered non-ABO. Immediately after the PTCA procedure while remaining patients at rest in the ward, 3-lead ECG was continuously registered for 12 hours (Philips Holter DigiTrak Plus recorder, Philips Medical Systems, Bothell, USA) at a sampling frequency of $200 \mathrm{~Hz}$.

The recordings were analyzed with Philips Zymed Holter series 1810 software, which classified the beats or events according to their origin, i.e., normal sinus, ventricular or supraventricular premature beats, QRS-like artifact and noise or change of signal quality. R-wave time positions and beat or event annotations were exported to a text file. R-R intervals were obtained and normal-to-normal $(\mathrm{N}-\mathrm{N})$ beats intervals were calculated from normal sinus depolarization. R-R interval from 
ectopic beats or atifacts were edited using a 5-beat sliding window algorithm, rejecting any beat that deviated more than a tolerance of $15 \%$ from the mean length of the preceding R-R intervals [5]. Rejected beats were replaced by the median of the previous $5 \mathrm{R}-\mathrm{R}$ intervals. ECG recordings and accuracy of R-wave detection were verified by visual assessment of the data.

\subsection{Holter and HRV analysis}

The 12-hour ECG recordings were displayed and analyzed by Philips Zymed Holter series 1810 software and then evaluated by 2 cardiologists, who were blinded to the patients' clinical data, in order to detect atrial arrhythmic events.

Two epochs were evaluated: (i) the first 10 minutes and (ii) the 12-hour period of R-R interval series after the PTCA procedure. ANS dynamics were evaluated by HRV analysis using standard time and frequency domain measures and the fractal-like index $\alpha_{1}$.

Heart rate (HR) mean, the standard deviation of normal $\mathrm{R}-\mathrm{R}$ intervals (SDNN) and the square root of the mean square differences of successive R-R intervals (rMSSD) were calculated as time domain indices for each epoch.

In order to compute standard frequency domain indices, R-R interval time series were pre-processed removing the mean value and resampling at $4 \mathrm{~Hz}$ after cubic spline interpolation. The power spectral density (PSD) of the processed R-R intervals of each patient was estimated by fitting an autoregressive (AR) model

$$
x(n)=-\sum_{k=1}^{n a} a_{k} x(n-k)+e(n)
$$

where $x(n)$ is the resampled R-R signal, $a_{k}$ are the model parameters, $e(n)$ is the model residual and $n a$ is the model order. Parameters of the model were estimated using the Burg method [6]. For 5-minute R-R interval segments a model order of 16 was used, following recommendation of previous studies [7]. For 12-hour R-R series the model order na was assessed using Broersen's combined information criterion (CIC) [8], chosen $n a=25$. from

The PSD of the modelled R-R signal was estimated

$$
P_{x x}^{A R}(f)=\frac{\sigma_{e}^{2}}{f_{s}\left|1+\sum_{k=1}^{n a} a_{k} e^{-j 2 \pi k f / f_{s}}\right|^{2}}
$$

where $\sigma_{e}^{2}$ is the model variance error, $0 \leq f \leq f_{S} / 2$ and $f_{s}$ is the sampling frequency $(f s=4)$. Frequency domain indices were calculated as the average power in the standard HRV frequency bands by integrating the PSD over those bands.

For the 12-hour period the following frequency indices were computed: very low frequency power (VLF: 0.003 $0.04 \mathrm{~Hz}$ ), low frequency power (LF: $0.04-0.15 \mathrm{~Hz}$ ) and high frequency power (HF: $0.15-0.4 \mathrm{~Hz}$ ).
Table 1. Basic patient characteristics.

\begin{tabular}{lcccc}
\hline & $\begin{array}{c}\text { All } \\
(\mathrm{n}=109)\end{array}$ & $\begin{array}{c}\text { ABO } \\
(\mathrm{n}=17)\end{array}$ & $\begin{array}{c}\text { non-ABO } \\
(\mathrm{n}=92)\end{array}$ & $\begin{array}{c}\mathrm{p} \\
\text { value* }\end{array}$ \\
\hline Age (y) & $65 \pm 10$ & $64 \pm 12$ & $66 \pm 10$ & 0.39 \\
Men/women & $85 / 24$ & $13 / 4$ & $72 / 20$ & 0.99 \\
Previous MI & $45(41 \%)$ & $8(47 \%)$ & $37(40 \%)$ & 0.56 \\
\hline ABO: atrial branch occlusion; *ABO vs non-ABO group, \\
MI: myocardial infarction.
\end{tabular}

For the first 10-minute period the frequency indices were: $\mathrm{LF}$ and $\mathrm{HF}$ power, $\mathrm{LF} / \mathrm{HF}$ ratio, normalized LF (LFnorm) and normalized HF (HFnorm). LFnorm and HFnorm were computed as 100 times LF or HF power divided by total power after subtracting VLF $(\leq 0.04 \mathrm{~Hz})$. Although it is still a subject of research, HF power is considered to be associated with parasympathetic activity, LF power with both sympathetic and parasympathetic reflexes $[9,10]$ and VLF is believed to reflect the activity of the renin-angiotensin and vagal systems [11].

Total and VLF power were computed on the entire recording of both 10-minute and 12-hour epochs. LF and $\mathrm{HF}$ power were calculated on consecutive 5-minute segments during both epochs and then averaged [12].

The fractal correlation properties were measured by the scaling exponent, $\alpha_{1}$, based on detrended fluctuation analysis (DFA) of the R-R signal, computed from the original short-term (from 4 to 11 beats) R-R interval [13]. This technique permits the detection of self-similar fluctuations included in an apparently non-stationary time series such as the R-R signal and provides short-term fractal correlation properties of heart rate dynamics.

\subsection{Statistical analysis}

Statistical differences were analyzed using the Student's t test for normally distributed data or by the Mann-Whitney $U$ tests (non-normal data). Due to markedly positive skewed distribution of VLF, LF and HF power, the natural logarithm (ln) transformations of these indices were used. Chi-squared test was used when comparing categorical data. A p-value $<0.05$ was considered statistically significant. All analyses were performed using SPSS for Windows, release 22.0 (SPSS Inc., Chicago, Illinois).

\section{Results}

No significant clinical differences were found between study groups, as described in Table 1 . Patients with ABO include 15 occluded branches arising from the right coronary artery and 2 emanating from the circumflex coronary artery. ABO patients showed greater values of plasma levels of high-sensitive troponin $\mathrm{T}$ than non-ABO group, reflecting atrial myocardial damage. 
Table 2. Arrhythmic and Holter findings: 12-hour Holter.

\begin{tabular}{|c|c|c|c|}
\hline & $\begin{array}{l}\mathrm{ABO} \\
\text { group } \\
(\mathrm{n}=17)\end{array}$ & $\begin{array}{c}\text { Non-ABO } \\
\text { group } \\
(\mathrm{n}=92)\end{array}$ & $\mathrm{p}$ value \\
\hline \multicolumn{4}{|c|}{ Arrhythmic findings, $\mathrm{n}(\%)$} \\
\hline Atrial arrhythmia & $7(41 \%)$ & $13(15 \%)$ & 0.020 \\
\hline Atrial fibrillation & $2(12 \%)$ & $0(0 \%)$ & 0.025 \\
\hline \multicolumn{4}{|c|}{ 12- hour HRV indices, mean $\pm \mathrm{SD}$} \\
\hline $\begin{array}{l}\text { Average HR } \\
\text { (beats/min) }\end{array}$ & $65.5 \pm 9.0$ & $63.3 \pm 9.5$ & 0.37 \\
\hline SDNN (ms) & $88.7 \pm 38.7$ & $89.8 \pm 30.0$ & 0.89 \\
\hline rMSSD (ms) & $30.4 \pm 20.5$ & $32.2 \pm 27.2$ & 0.75 \\
\hline $\ln \operatorname{VLF}\left(\mathrm{ms}^{2}\right)$ & $7.54 \pm 0.98$ & $7.66 \pm 0.81$ & 0.58 \\
\hline $\ln \mathrm{LF}\left(\mathrm{ms}^{2}\right)$ & $5.35 \pm 1.15$ & $5.47 \pm 1.02$ & 0.67 \\
\hline $\ln \mathrm{HF}\left(\mathrm{ms}^{2}\right)$ & $4.88 \pm 1.14$ & $5.07 \pm 1.06$ & 0.50 \\
\hline$\alpha_{1}$ & $1.07 \pm 0.32$ & $1.10 \pm 0.25$ & 0.63 \\
\hline
\end{tabular}

\subsection{Atrial arrhythmic findings}

As summarized in Table 2, 12-hour Holter ECG analysis found that atrial arrhythmia (41\% vs. $15 \%$, $\mathrm{p}=0.02$ ) and atrial fibrillation episodes ( 2 vs. $0, \mathrm{p}=0.025$ ) were more frequently encountered in $\mathrm{ABO}$ patients compared to non-ABO. Atrial arrhythmia events were considered when 3 to 20 ectopic beats with an average frequency of 125 beat per minute were found. Atrial fibrillation episodes in the two $\mathrm{ABO}$ patients lasted less than 10 seconds before reverting to sinus rhythm [4].

\subsection{HRV after PTCA}

During the first 10 minutes immediately after PTCA (Table 3), SDNN was significantly lower in ABO patients respect to non- $\mathrm{ABO}(32.5 \pm 16.0$ vs. $49.5 \pm 28.6 \mathrm{~ms}, \mathrm{p}=$ 0.01 ), showing a reduced autonomic response. RMSSD, $\mathrm{LF}$ and $\mathrm{HF}$ indices were lower in the $\mathrm{ABO}$ group but without statistical significance. The average heart rate (HR), normalized LF and HF values, LF/HF ratio and fractal DFA index $\alpha 1$ were similar in both groups.

Table 2 shows HRV indices computed in the 12-hour period of R-R interval series after the PTCA procedure. No significant differences were found between $\mathrm{ABO}$ and non-ABO groups. The HRV values tend to recover after several minutes of the procedures since no significant differences were found between $\mathrm{ABO}$ and non- $\mathrm{ABO}$ groups when evaluated the 12-hour period.

\section{Discussion}

The principal finding of this study is that a significant reduction of the autonomic response was observed in patients with accidental atrial branch occlusions after PTCA procedures, as shown by the SDNN values of R-R interval series measured during 10 minute period immediately after the procedures.

Table 3. HRV findings: first 10min after PTCA.

\begin{tabular}{lccc}
\hline & $\begin{array}{c}\text { ABO } \\
\text { group } \\
(\mathrm{n}=17)\end{array}$ & $\begin{array}{c}\text { Non-ABO } \\
\text { group } \\
(\mathrm{n}=92)\end{array}$ & $\mathrm{p}$ value \\
\hline \multicolumn{4}{l}{ First 10-min HRV indices, mean \pm SD } \\
\hline $\begin{array}{l}\text { Average HR } \\
\text { (beats/min) }\end{array}$ & $66.9 \pm 10.1$ & $64.1 \pm 11.4$ & 0.35 \\
SDNN (ms) & $32.5 \pm 16.0$ & $49.5 \pm 28.6$ & 0.011 \\
rMSSD (ms) & $27.0 \pm 20.6$ & $31.9 \pm 27.0$ & 0.34 \\
ln LF (ms $\left.{ }^{2}\right)$ & $4.88 \pm 1.49$ & $5.44 \pm 1.39$ & 0.13 \\
LFnorm & $55.1 \pm 25.7$ & $56.9 \pm 19.6$ & 0.74 \\
ln HF $\left(\mathrm{ms}^{2}\right)$ & $4.43 \pm 1.17$ & $4.95 \pm 1.31$ & 0.12 \\
HFnorm & $34.6 \pm 17.9$ & $36.3 \pm 15.0$ & 0.67 \\
LF/HF & $2.53 \pm 2.07$ & $2.28 \pm 1.81$ & 0.61 \\
$\alpha_{1}$ & $0.96 \pm 0.43$ & $1.06 \pm 0.28$ & 0.22 \\
\hline
\end{tabular}

The reduced number of patients in the $\mathrm{ABO}$ group is a significant limitation to obtain statistically significant inference. The indices related to the sympathetic and parasympathetic responses did not provide significant differences between the study groups. However, there is a tendency for a higher HR and a lower vagal response, as shown by the HF and rMSSD indices, in the ABO group.

As found in previous study [4], atrial myocardial ischemic damage after $\mathrm{ABO}$ may be linked with atrial fibrillation and atrial arrhythmic episodes. It is well documented that a reduced HRV was found in patients recovering from myocardial infarction and, further, patients with smaller HRV have worse cardiac death prognostic [14,15]. A reduced autonomic response described by SDNN in the ABO group and a tendency of a reduction in vagal tone reflected by rMSSD and HF indices may infer a relationship between atrial myocardial ischemia and reduced vagal activity.

Studies assessing the effect of considerably long coronary artery balloon occlusions during PTCA on ANS response found a significant reduction of the vagal activity, measured by HF power, during the occlusion of right coronary arteries [2].

Since an important number of patients undergoing elective percutaneous interventions of the right or circumflex coronary arteries are at risk of suffering accidental occlusion of coronary atrial branches, technical or procedural actions to protect these branches should be implemented in those patients.

\section{Conclusion}

Acute atrial ischemia can lead to a global decrease in the autonomic response that recovers after several minutes. Besides, atrial myocardial ischemic damage following an accidental atrial branch occlusion may be related to atrial 
fibrillation and atrial arrhythmic episodes.

\section{Acknowledgements}

This work was supported by TEC2014-60337-R grant from the Ministerio de Economía y Competitividad (MINECO).

\section{References}

[1] Cinca J, Janse MJ, Moréna H, Candell J, Valle V, Durrer D. Mechanism and time course of the early electrical changes during acute coronary artery occlusion. An attempt to correlate the early ECG changes in man to the cellular electrophysiology in the pig. Chest. 1980;77:499-505.

[2] Gomis P, Caminal P, Vallverdú M, Warren SG, Stein PK, Wagner GS. Assessment of autonomic control of the heart during transient myocardial ischemia. J Electrocardiol 2012;45(1):82-89.

[3] Álvarez-García J, Vives-Borrás M, Ferrero A, Aizpurua DA, Peñaranda AS, Cinca J. Atrial coronary artery occlusion during elective percutaneous coronary angioplasty. Cardiovasc Revasc Med. 2013;14:270-4.

[4] Álvarez-García J, Vives-Borrás M, Ferrero A, Gomis P, Ordoñez-Llanos J, Ferrero-Gregori A, Serra-Peñaranda A, Cinca J. Electrophysiological Effects of Selective Atrial Coronary Artery Occlusion in Humans. Circulation. 2016;133:2235-2242.

[5] Clifford GD, McSharry PE. Tarassenko L. Characterizing Artefact in the Normal Human 24-Hour RR Time Series to Aid Identification and Artificial Replication of Circadian Variations in Human Beat to Beat Heart Rate using a Simple Threshold. Computers in Cardiology 2002;29:129132.

[6] Therrien ChW. Discrete Random Signals and Statistical Signal Processing. Englewood Cliffs, New Jersey:
[7] Boardman A, Schlindwein FS, Rocha AP, Leite A. A study on the optimum order of autoregressive models for heart rate variability. Physiol Meas 2002;23:325-336.

[8] Broersen, PMT. Finite sample criteria for autoregressive order selection, IEEE Trans Signal Processing 2000;48: 3550-8.

[9] Malliani A. Heart reate variability: from bench to bedside. Eur J Intern Med 2005;16:12.

[10] Kleiger RE, Stein PK, Bigger JT Jr. Heart rate variability: measurement and clinical utility. ANE. 2005; 10: 1-14.

[11] Taylor JA, Carr DL, Myers CW et al. Mechanisms underlying very-low-frequency RR-interval oscillations in humans. Circulation 1998; 98: 547-55.

[12] Stein PK, Sanghavi D, Sotoodehnia N, et al. Association of Holter-based measures including $\mathrm{T}$-wave alternans with risk of sudden cardiac death in the community-dwelling elderly: The Cardiovascular Health Study. J Electrocardiol 2010;43:251-259.

[13] Peng CK, Havlin S, Stanley HE, Goldberger AL. Quantification of scaling exponents and crossover phenomena in nonstationary heartbeat time series. Chaos 1995;5:82.

[14] Kleiger RE, Miller J P, Bigger JT, Moss AJ. Decreased heart rate variability and its association with increased mortality after acute myocardial infarction. The American Journal of cardiology, 1987;59(4):256-262.

[15] Bigger JT. The predictive value of RR variability and baroreflex sensitivity in coronary heart disease. Cardiac Electrophysiology Review, 1997;1(1):198-204.

Address for correspondence.

Pedro Gomis.

Universitat Politècnica de Catalunya,

Pau Gargallo, 14, ESAII, 08028 Barcelona, Spain. pedro.gomis@upc.edu. 